\title{
Estrategias pedagógicas para la motivación lectora a través de un software de lectura interactiva
}

\section{Pedagogical Strategies for Reading Motivation Through an Interactive Reading Software}

\author{
César Augusto Vásquez*, Amparo Romero y Ruth Yamile Molina** \\ Fecha de recepción: 15 de julio de 2009 \\ Fecha de aprobación: 31 de agosto de 2009
}

\section{Resumen:}

La ponencia presenta el diseño y la construcción de un módulo de estrategias pedagógicas para la motivación de lectura de textos narrativos en el grado Transición del Colegio Liceo Miguel de Cervantes Saavedra, del municipio de Fusagasugá (Cundinamarca). El módulo pedagógico tiene como finalidad motivar la lectura a través de guías complementarias y creativas para la aplicación del software educativo Lectura interactiva, elaborado por Olga Patricia Parra (2000).

Palabras clave: estrategia pedagógica, módulo, lectura, lectura interactiva, software.

Investigador y docente, Universidad Santo Tomás. Correo electrónico: cesarvasquez@correo.usta.edu.co

** Investigadoras asociadas de la Institución Educativa Distrital Montebello y de la Institución Educativa Departamental San Gabriel. (Bogotá-Colombia). Correos electrónicos: ruyalmoj@yahoo.es, amaparcy@yahoo.es 


\section{Abstract}

The paper presents the pedagogic strategies of a module, its design and construction to motivate the reading of narrative texts at Colegio Liceo Miguel de Cervantes Saavedra, transition grade in Fusagasugá-Cundinamarca. The pedagogic module aims at reading's motivation through complementary and creative guides for the application of the education's software "Interactive Reading" created by Olga Patricia Parra (2000).

Key words: pedagogic strategy, module, reading, interactive reading, software.

\section{Introducción}

Existe una idea reiterada sobre el concepto de lo que es saber leer: pasar la vista por los signos de la palabra escrita para interpretar el sentido de los textos. Sin embargo, el concepto de lectura se ha ampliado debido a factores socioculturales, como la incorporación en los procesos educativos de las Nuevas Tecnologías de la Información y la Comunicación (NTIC).

Por texto, palabra proveniente de la lengua griega que significa tejido, se aborda hoy en día toda clase de construcción natural y cultural que se puede destejer, es decir, deshilar hilo a hilo para poder ser leído y, por tanto, interpretado. Por ejemplo, una construcción cultural como una película cinematográfica es un texto en la medida en que se pueden leer cada uno de los hilos que la constituyen: la historia o argumento, el tiempo, la música, la escenografía o el espacio, la fotografía, los personajes, etc. En este sentido, como lo explica Vásquez G. (2000), el concepto tradicional de lectura es explotado en beneficio del desciframiento de signos, no solamente escritos, sino también de signos orales, icónicos, audiovisuales y multimediales.

Es innegable que cuando los niños y niñas llegan por primera vez a la vida escolarizada, sea ésta vivida en el jardín infantil de párvulos, en preescolar o en la escuela primaria, poseen de antemano un andamiaje sensoriomotor mínimo que les hace ser capaces de enfrentar el mundo externo. De otra forma, no se explican que puedan socializarse con los adultos y los demás niños. Dadas estas condiciones sensoriomotoras, el niño y la niña llegan preparados al ámbito escolarizado en el que se espera logren desarrollar sus competencias sociales, comunicativas y cognitivas de la mejor manera. No obstante, muchos de estos niños no logran satisfactoriamente desarrollar a cabalidad estas competencias, ¿a qué se debe?

Los estudios psicológicos demuestran permanentemente que los dispositivos pedagógicos, para activar y desarrollar las capacidades sensoriomotoras y cognitivas de los niños, están desfasados en relación con las necesidades de los infantes, pues todavía se cree en el 
ámbito educativo que el centro de la acción pedagógica es el pensamiento lógico-racional, desconociendo otros tipos de pensamiento como el lingüístico en sus formas orales, el musical, el espacial y el cinestésico-corporal.

En la presente propuesta se pretende rescatar a nivel pedagógico estos y otros tipos de pensamiento, especialmente, el que corresponde al lingüístico, en sus manifestaciones orales, las cuales involucran los niveles fonéticos (articulación y producción de la voz), kinésico o quinésico (movimientos y gestos corporales) y proxémico (movimiento y traslación del cuerpo en el espacio).

A partir de cada uno de estos niveles creemos que es necesario redimensionar el campo de la lectura, pues es en el nivel oral en el que comienza el trabajo pedagógico de destejer el texto que se llama mundo, casa, escuela, aula, por medio de instrumentos o herramientas interactivas que, como las tecnológicas, acercan los niños y niñas a los textos y al mundo de la lectura.

\section{Objetivos}

\section{Objetivo general}

Presentar el diseño e implementación de un módulo didáctico y pedagógico para mediar los procesos de enseñanza y aprendizaje de la lectura de textos narrativos en estudiantes de grado Transición del Liceo Miguel de Cervantes Saavedra a través de un software educativo de lectura interactiva.

\section{Objetivos específicos}

- Propiciar ambientes pedagógicos adecuados para la formación de niños lectores de textos desde los criterios de comunicabilidad, interactividad, sociabilidad y dialogicidad, propios de la mediación pedagógica.

- Utilizar adecuadamente con fines pedagógicos las NTIC para desarrollar propuestas no convencionales de enseñanza y aprendizaje de la lectura.

\section{Desarrollo}

Desde los anteriores planteamientos, la propuesta está orientada a desarrollar alternativas pedagógicas y metodológicas para acercar al estudiante de Transición del Liceo Miguel de Cervantes Saavedra, a la lectura de textos narrativos, utilizando herramientas tecnológicas como el software educativo que ampliaría el espectro de la comprensión antes que la decodificación lectora.

La propuesta se sustenta en el enfoque de la investigación descriptiva de carácter cualitativo, desde donde se observó, describió, analizó e interpretó la necesidad de aplicar estrategias pedagógicas en la utilización de las NTIC para la motivación lectora de textos narrativos en los estudiantes grado transición del Liceo Miguel de Cervantes Saavedra, del municipio de Fusagasugá (Cundinamarca).

En ese sentido, esta investigación se caracteriza por la observación y descripción de lo que los estudiantes hicieron, cómo se comportaron 
y cómo interactuaron entre sí, para describir sus relaciones con las NTIC en el transcurso del desarrollo de la propuesta. Se utilizó la inferencia inductiva y el análisis sucesivo de los datos a través del tiempo con criterios de credibilidad, transferibilidad y estudio, que se realizó por medio de la recolección de datos y de los instrumentos de investigación cualitativa (observación, encuestas, entrevistas, diario de campo, fotografías, videos y talleres pedagógicos).

La intervención pedagógica que se realizó en el Liceo Miguel de Cervantes Saavedra, partió del análisis de las necesidades de la población en estudio, es decir, los estudiantes, quienes nos Ilevaron a apreciar que las NTIC aplicadas al proceso de aprendizaje de la lectura, dinamiza el quehacer pedagógico de los docentes en esta Institución. La intervención en esta población se enriqueció con la utilización de las NTIC a través de propuestas de prácticas pedagógicas para hacer de las clases experiencias didácticas y creativas. Por ello se diseñó y elaboró un módulo como estrategia pedagógica para la motivación de la lectura de textos narrativos a través de un software interactivo, en el cual los estudiantes fueron el centro del proceso pedagógico.

Para tales fines se organizaron algunas actividades con el propósito de crear situaciones didácticas a través de las NTIC, en las que intervino la creatividad de los docentes, no sólo en el diseño de actividades, sino también en la variación y adecuación de estrategias pedagógicas, según las necesidades de la población estudiantil. Es relevante mencionar que la selección de actividades como talleres, charlas, conversatorios permitieron el logro de los objetivos propuestos y, por ende, el desarrollo de la capacidad lectora de los niños y niñas de grado transición a través de las NTIC.

Los docentes del Liceo Miguel de Cervantes Saavedra, como grupo humano y promotor del saber académico en la Institución, construyen variedades de estrategias pedagógicas, actividades y modelos didácticos que les permiten ver las dificultades en el proceso enseñanzaaprendizaje en cada una de las asignaturas y áreas del plan de estudio. En ese sentido, el docente es quien crea condiciones, diseña actuaciones, detecta contradicciones, hace explícitas las relaciones, facilita materiales de trabajo y participa en la evaluación junto con los estudiantes. En definitiva, es el encargado de orientar el proceso pedagógico, interpretando las respuestas de los niños y ayudándoles a construir sus propios aprendizajes.

Sin embargo, los docentes del Liceo Miguel de Cervantes Saavedra cada día se ven enfrentados a un reto mayúsculo: deslegitimar la concepción mecanicista e instrumental que enfatiza en la transmisión de información, en aras del desarrollo de estrategias para la construcción de conocimiento soportada en las NTIC. EI nivel de exigencia de este nuevo escenario para la institución educativa requiere necesariamente que desde las edades más tempranas de la vida de los niños se inicie un intenso proceso de formación y desarrollo de competencias básicas, el cual, de no iniciarse de manera oportuna, sencillamente estas competencias no se desarrollarán de manera adecuada. 
En consecuencia, la explosión de la información y los avances tecnológicos le plantean nuevos desafíos a los docentes del Liceo Miguel de Cervantes Saavedra, institución en la cual el problema no es el aumento y el almacenamiento de la información, sino la creación de una nueva mentalidad para participar del desarrollo del conocimiento, la ciencia y la tecnología. Por ello, la investigación, llevada a cabo durante un año de trabajo con la comunidad de docentes, directivos, padres de familia y estudiantes en la Institución, se orientó a brindar herramientas tecnológicas para abordar la habilidad básica para el acceso a la información y, posteriormente, a la construcción del conocimiento: la lectura. Así, la lectura se ve exigida a redefinir su propia función dentro del trabajo escolar, independientemente de las áreas disciplinares o de los saberes construidos, a partir de la mediación de las nuevas tecnologías que atraviesan de punta a punta los enfoques pedagógicos y didácticos que sustentan las prácticas docentes. Se trató, entonces, de establecer la relación entre lenguaje, nuevas tecnologías y competencias comunicativas.

Con base en estos parámetros metodológicos, la investigación se llevó a cabo con dieciocho estudiantes, quienes se caracterizan por pertenecer a una población de estrato socioeconómico medio, cuyas edades oscilan entre cinco y seis años y estudian en el grado transición del Liceo Miguel de Cervantes Saavedra, del municipio de Fusagasugá (Cundinamarca).

A su vez, con el propósito de hacer un desarrollo metodológico en profundidad se eligió el método etnográfico como la alternativa con más potencial en la investigación social, en razón de la propuesta educativa que nos convoca. La etnografía es un método de investigación que parte de la idea de asumir la necesidad de una inmersión en la realidad investigada, contando para ello con herramientas básicas como observaciones, entrevistas, encuestas, talleres pedagógicos, fotografías, conversaciones informales y diario de campo. Estas herramientas permitieron capturar la información necesaria para la posterior sistematización de dicha información en el ejercicio investigativo, así como para el diseño del módulo y el análisis de resultados que se dieron.

A continuación, se describen los instrumentos proporcionados por el método etnográfico que se aplicaron para el desarrollo de la investigación descriptiva de carácter cualitativo:

a) Observación: es una técnica fundamental del proceso investigativo cualitativo, en el cual los investigadores se apoyaron para obtener el mayor número de datos que sirvieron para un posterior análisis. Este ejercicio, realizando durante seis meses en el Liceo Miguel de Cervantes Saavedra, nos sumergió en la realidad educativa de los estudiantes participantes, lo que significó observar el proceso de enseñanzaaprendizaje empleado por los docentes de la institución educativa. Esta estrategia investigativa permitió registrar periódicamente comportamientos, sentimientos, inquietudes, facilidades o dificultades de los estudiantes en relación con el uso de las NTIC en sus prácticas pedagógicas. 
Para realizar la observación se tuvieron en cuenta los siguientes aspectos: determinación del propósito de la observación, situación del caso observado, formulación de los objetivos de las observaciones, forma de registrar la información, observación crítica y cuidadosa, registro de datos observados, análisis e interpretación de datos, elaboración de conclusiones e informe de la observación. Asimismo, los recursos empleados para la observación fueron: fichas, record de anécdotas de estudiantes y docentes, grabaciones, fotos y diario de campo.

La observación se realizó teniendo en cuenta los siguientes aspectos: observación directa, participante, de campo, de grupo y estructurada. Como investigadores pasamos el mayor tiempo en el lugar donde ocurrieron los hechos y los fenómenos investigados. Esta situación nos permitió la comprensión de los procesos de enseñanza-aprendizaje soportados en las NTIC.

b) Talleres pedagógicos: fueron las herramientas que nos permitieron llevar a cabo con los estudiantes, actividades de motivación de lectura de textos narrativos mediante el módulo diseñado. Los talleres fueron el resultado de integrar la teoría y la práctica en un trabajo colectivo, en el que se apreció la utilización de las NTIC como herramientas que facilitan la comunicación y la aprehensión de dispositivos novedosos que posibilitan el acceso a la información y generan rituales distintos para la construcción del conocimiento. Por ello, los talleres pedagógicos que se realizaron resultaron ser una vía idónea para formar, desarrollar y perfeccionar hábitos, habilidades y capacidades de los estudiantes frente a las NTIC, lo que les permitió operacionalizar las propuestas tecnológicas, en especial con la aplicación del software educativo de lectura interactiva.

c) Encuesta: es una técnica cuantitativa que permitió la recolección de información realizada sobre una muestra del grupo de docentes, en la que se precisó utilizar procedimientos estandarizados de interrogación, con el fin de conseguir mediaciones cuantitativas sobre una gran cantidad de características objetivas y sujetivas, en relación con la utilización de las NTIC en los procesos pedagógicos. Además, permitió obtener información sobre los conocimientos, usos benéficos y ventajas que ofrecen las NTIC en el ejercicio pedagógico.

d) Entrevistas: las entrevistas generalmente se emplearon para recoger información que no se pudo obtener a través de la observación y, a su vez, para verificar los datos obtenidos en las observaciones. En el caso del presente proyecto de investigación, el objetivo de las entrevistas fue explicar el punto de vista de los participantes: cómo pensaron, interpretaron y explicaron su comportamiento en el contexto natural de la acción pedagógica en relación con las NTIC. Las entrevistas se llevaron a cabo de forma individual y grupo, en las cuales los investigadores tuvimos tres opciones para recoger los datos: tomar notas durante la entrevista, tomar notas después de ella o grabarla en cassette. En el caso particular 
de los directivos docentes, se les entregó con un día de anticipación los cuestionarios para que se familiarizaran con la temática de la entrevista.

Los tipos de entrevistas que se realizaron fueron la no estructurada, entrevista a profundidad, enfocada y focalizada. Las preguntas o guiones se elaboraron antes de realizar las entrevistas, lo que permitió modificar el orden de las preguntas, la forma de encausar las preguntas, adaptarlas a las diversas situaciones y características particulares de los entrevistados (estudiantes, directivos, docentes y expertos en NTIC).

La entrevista a profundidad se realizó cuando se entrevistó al doctor Luis Facundo Maldonado Granados, investigador experto en NTIC aplicadas a la educación; de igual forma se utilizó cuando se entabló conversación con los docentes investigadores de la Universidad Santo Tomás, Carlos Bernal y Julio Rojas. A través de estas entrevistas se pudieron obtener sus apreciaciones personales y profundas sobre el manejo, la importancia y el uso de las NTIC en el ejercicio pedagógico del docente. Por otro lado, se evidenció la participación y la aprehensión de la temática por parte de los entrevistados y entrevistadores, de esto se obtuvo una serie de conclusiones fundamentales y significativas acerca de las NTIC en las nuevas dinámicas socio-culturales que influyen en la construcción del conocimiento escolar.

La entrevista focalizada se desarrolló cuando los estudiantes, docentes y directivos del Liceo Miguel de Cervantes Saavedra y expertos contestaron preguntas en grupo. Los entrevistados generaron reflexiones y análisis, que la entrevista individual no ofreció, hecho que llevó a la confrontación de diversas opiniones y puntos de vista sobre el manejo, utilidad y aprovechamiento de las NTIC. Este ejercicio permitió evidenciar el manejo, acceso, conocimiento, expectativas, fortalezas y debilidades de los docentes sobre el uso y aprovechamiento de las Nuevas Tecnologías de la Información y la Comunicación en el campo educativo. Las entrevistas que se desarrollaron en el Liceo Miguel de Cervantes Saavedra y en la Universidad Santo Tomás fueron caracterizadas por un buen ambiente, camaradería y empatía de trabajo.

e) Fotografías: la fotografía es un lenguaje, una técnica y un arte. Es un modo de captar y exponer gráficamente la realidad visual. Cada foto es un fragmento, un recorte de una realidad interpretada con nuestra mirada a través de la cámara. En el desarrollo de la aplicación de la investigación, se utilizó la fotografía como medio para identificar la forma, el color, las cualidades, las habilidades, las destrezas y el mensaje que había en cada hecho, en cada objeto, en cada instante del proceso investigativo.

En esta investigación fue muy importante capturar cada imagen, saber mirar y escoger el momento preciso para plasmar y obtener los mejores resultados. Además se requirió un cierto tiempo de exposición, enfoque $y$ determinados elementos por tener en 
cuenta para recoger todo aquello que se tiene en el diario de campo. Las fotos que se tomaron en el desarrollo de las distintas actividades se asumen como una fuente de documentación, interpretación, memoria histórica de investigación, de análisis, de recuerdos y experiencias vividas para hallar las fortalezas y las debilidades en cada una de las acciones realizadas como: las entrevistas a docentes y a la rectora de la institución, en la aplicación de la propuesta con los niños y niñas de transición, en las visitas a las dependencias del colegio y en el estudio colectivo e individual de cada uno de los que participaron en este proceso.

f) El diario de campo: sirvió como instrumento para el proceso de investigación que se adelantó en el Liceo Miguel de Cervantes Saavedra, en el cual la información que se obtuvo se registró en un cuaderno diseñado para ello. Las hojas de este diario se encuentran divididas en dos secciones: en la primera se encuentran las observaciones y en la segunda las impresiones o conclusiones, producto de la observación general, registros del lugar, fecha y hora de la observación, diferentes acciones de los docentes y agentes externos como olores, sonidos, clima y otros.

El diario de campo, como herramienta etnográfica en este proceso de investigación cualitativa, promovió reflexiones sistemáticas sobre la información registrada. En cada sesión de observación se pretendió centrar la atención en un objetivo particular, lo que requirió de un protocolo o listado con los aspectos que guían la observación como las siguientes: ¿a quién se observa?, ¿cuántos estudiantes hay en el ejercicio pedagógico? y ¿quiénes son?, ¿qué actividades realizan?, ¿cómo se desarrolla la sesión?, ¿cuál es la naturaleza del ejercicio pedagógico?, ¿qué dicen o hacen los participantes?, ¿cómo es el contexto físico o ambiente?, ¿cómo es el clima de la sesión, qué actitudes o valores emergen?, ¿cómo termina la sesión? ¿el grupo está unido?, ¿dividido?, ¿el informante está cansado?, ¿aburrido?, ¿qué actividades o interacciones resultan significativas para el esclarecimiento del problema?, etc.

Se realizó un registro general, teniendo en cuenta lugares, fechas y horas del momento de la observación, escribiendo todo lo observado y percibiendo las acciones, los movimientos, los contextos y las impresiones que estos elementos causaron en el desarrollo de la investigación.

El diario de campo proporcionó datos reflejados en descripciones detalladas de los fenómenos observados, junto con las interpretaciones de los investigadores, que al mismo tiempo, constituyeron datos brutos que luego sirvieron de análisis para dar respuesta al problema de la investigación. Para el desarrollo de la actual investigación, las notas del diario de campo fueron de dos tipos:

1. Descriptivas: se tuvieron en cuenta dibujos, palabras, planos, diagramas, etc., como formas de expresión de lo que ocurrió en el contexto, por ejemplo, descripciones del 
entorno, de la apariencia física y del carácter de los participantes, de lo que dijeron y de cómo actuaron.

2. Reflexivas: las valoraciones incluyeron temas emergentes y patrones de respuesta, pensamientos acerca de los problemas observados, preocupaciones metodológicas, conceptuales, éticas, etc. Además, se utilizó el diario de campo como espacio reflexivo cuando se especuló sobre sentimientos, interpretaciones, ideas e impresiones que se formaron a partir de los datos que se fueron tomando.

Por último, es necesario recordar que la investigación tiene como objetivo el "diseño e implementación de un módulo como estrategia pedagógica para la motivación lectora de textos narrativos a través de un software de lectura interactiva. No de otra forma se justifica la existencia de todos los elementos teóricos y metodológicos previamente analizados.

El concepto de "módulo" ha terminado desvirtuado, al ser considerado simplemente como un "libro" o texto de carácter "sagrado" y "cerrado", lo que invalida su esencia, puesto que su concepción modular obliga a pensar en una estrategia pedagógica capaz de soportar la inclusión y la exclusión de información, según las necesidades $\mathrm{y}$, sobre todo, flexible frente a la posibilidad de construcción de conocimiento.

Desde esta perspectiva, el módulo en la investigación, es una herramienta multimedial, o por denominarlo de otra forma, es un "kit mediático" que da cuenta de un equipo o caja de herramientas en la idea de construir un juego de armar con diferentes medios: texto impreso en formato de cartilla, en el cual se ubican talleres didácticos, ejercicios de aplicación y evaluaciones pedagógicas, para que los docentes desarrollen estrategias tecnológicas con la finalidad de enseñar procesos de lectura; y un CD-ROM que contiene las orientaciones pedagógicas y metodológicas del texto impreso y que tiene como finalidad complementar las actividades propuestas por la cartilla.

El módulo Huellitas mágicas de motivación lectora de textos narrativos para grado transición tiene como finalidad presentar, secuenciar, relacionar, apoyar, complementar, graduar, interactuar, los contenidos de cuatro unidades que conforman la propuesta global de lectura.

El proceso de elaboración del diseño del módulo tuvo en cuenta condiciones tales como: adaptarse a las condiciones e intereses de los docentes y estudiantes de grado transición; incorporar estrategias de lectura pertinentes y basar su diseño en principios de orden pedagógico y didáctico. El objetivo fue, en definitiva, diseñar una herramienta pedagógica multimedial para motivar a los niños y niñas a leer textos narrativos, de aproximadamente 80 páginas de extensión, con pertinencia pedagógica, dinamismo, creatividad y asociación cognitiva con el software educativo.

Dentro del módulo, las guías de lectura y los talleres pretendieron facilitar la interacción de los nuevos conocimientos, para garantizar la asimilación y la integración de la información ofrecida posibilitando los procesos de apropiación comprensiva de esta información por 
parte de los estudiantes. Las guías de lectura y los talleres fueron las estrategias pedagógicas que orientaron las actividades que están en el software educativo Lectura interactiva, lo cual propició procesos cognitivos, comunicativos y valorativos de los estudiantes, con el fin de que puedan trabajarlos de manera autónoma.

Los aspectos considerados al elaborar las guías de lectura y los talleres fueron los siguientes:

a) Orientaciones claras de las tareas, procesos, situaciones y actividades por desarrollar en el proceso de la lectura.

b) Descripciones específicas para hacer que la interpretación sea más agradable para el estudiante.

c) Rutas de navegación que dan cuenta de los procesos cognitivos, comunicativos, valorativos e interpretativos para crear seguridad en el proceso de enseñanza.

d) Estrategias didácticas en las diferentes actividades.

e) Articulación de los contenidos del software educativo Lectura interactiva, con los contenidos de la cartilla.

f) Presentación de los contenidos, tanto en el software como en la cartilla, en forma ordenada, consistente y pertinente, facilitando el aprendizaje, orientando y animando a los estudiantes para continuar el proceso de la enseñanza de la lectura.

El módulo pedagógico para la motivación lectora, didácticamente presenta unas características especiales que lo diferencian de otros instrumentos para la enseñanza. A continuación, se presentan los aspectos que se consideran fundamentales en un módulo pedagógico de este tipo:

- Introducción

- Propósitos

- Conceptualización

- Etapa inicial

- Etapa de progreso

- Etapa de afianzamiento

- Unidad

- Unidad 2

- Unidad 3

- Unidad 4

A su vez, cada unidad contiene los siguientes elementos: objetivo, actividades, mapa conceptual y propuesta evaluativa por competencias.

\section{Conclusiones}

En la actualidad, conocer y utilizar la tecnología no constituye un privilegio, por el contrario, es una necesidad debido al avance de la ciencia y la construcción de nuevos saberes y conocimientos, al convertirse en un factor determinante en los niveles de eficiencia y competitividad a nivel personal, profesional y empresarial. Sin embargo, en el contexto de la educación, la utilización de las nuevas tecnologías es un aspecto en discusión, debido a que las prácticas pedagógicas que se han desarrollado al amparo de la "tecnología educativa". Hasta el momento, se caracterizan por "la fragmentación 
del conocimiento y la poca utilidad del mismo para generar procesos de mejora y cambio educativo" (Area M., 2001, p. 160)

En este comienzo de siglo estamos asistiendo a un cuestionamiento y replanteamiento de las áreas de conocimiento, de las disciplinas, de las formas de organizar y abordar el conocimiento científico. La superespecialización, la fragmentación de la realidad pedagógica en parcelas disciplinares distantes está dificultando y entorpeciendo la elaboración de respuestas globales ante los nuevos problemas educativos generados en las sociedades de la información, en las cuales la formación escolar es la primera afectada.

En gran parte de las instituciones educativas de nuestro sistema escolar colombiano, ya sean urbanas o rurales, el desarrollo y puesta en práctica del currículo en las aulas se realiza mediante una tecnología monomediada (casi siempre de naturaleza textual), no desarrollando suficientemente experiencias de aprendizaje sobre variadas tecnologías y formas expresivas de la información. Por ejemplo, en la formación escolar básica en el contexto del Liceo Miguel de Cervantes Saavedra, la aparición y el desarrollo de la informática ha abierto la formación a otro tipo de tecnologías que pretenden complementar la información textual ubicada en los libros tradicionalmente utilizados para el aprendizaje de los estudiantes, lo que ha implicado un reto para los docentes, ya que los procesos de enseñanza-aprendizaje en la institución escolar se desarrollan en su mayoría con una tecnología inventada en el siglo XV, estando ausentes los medios, artefactos y lenguaje inventados en el siglo XX (cine, televisión, video, programas informaticos).

Este fenómeno implica, entre otros factores, los siguientes: a) la formación escolar alfabetiza preferentemente en la lecto-escritura por lo que el estudiante desarrolla casi exclusivamente un tipo de habilidades de decodificación simbólica en detrimento de otros sistemas de información y comunicación, y b) la escuela fundamentalmente cultiva y legitima la cultura impresa, pero desconoce otras manifestaciones culturales propias de la esfera audiovisual. De esta manera, el estudiante se enfrenta en su proceso de formación a por lo menos dos tipos de culturas que se le presentan separadamente: la cultura del "afuera" de la escuela, que es audiovisual e informática y la cultura "impresa" de la escuela.

En definitiva, como lo argumenta Area Moreira en el artículo ya referenciado, el problema que se está enunciando hace referencia a que en el currículo actualmente desarrollado en nuestras escuelas, se encuentran ausentes procesos de alfabetización audiovisual e informática. Nuestro profesorado y consecuentemente sus prácticas docentes obvian el desarrollo de una alfabetización en los alumnos en el campo de la imagen y los sistemas. Las razones que subyacen a este problema son muchas, entre las que podemos destacar la falta de formación del profesorado en este campo, ausencia de suficientes recursos audiovisuales e informáticos en las escuelas, desconocimiento del uso e integración curricular de estas tecnologías, cambios en la organización y modos de presentación del conocimiento al alumnado, 
pero sobre todo, temores y desconfianzas del profesorado ante éstas.

\section{Referencias}

Angarita, H. (Comp.) (2001). Pedagogía y Educación a Distancia. Bogotá: Universidad Nacional de Colombia Abierta y a Distancia.

Bonilla, E. \& Rodríguez, P. (2002). Más allá de los dilemas de los métodos: la investigación en ciencias sociales. Bogotá: Editorial Norma.

De Zubiria, S. (1999). Teoría de las seis lecturas. Bogotá: Alberto Merani.

Delgado, J.M. \& Gutiérrez, J. (1999). Métodos y técnicas cualitativas de investigación en ciencias sociales. Madrid: Síntesis.

Ferreiro, E. \& Teborosky, A. (1972). Los sistemas de escritura en el desarrollo del niño. Madrid: Siglo XXI Editores.
Ferreiro, E. \& Teberosky, A. (1979). Los sistemas de escritura en el desarrollo del niño. México: Siglo XXI Editores.

Ferrés, J. \& Márquez, P. (1999). Comunicación educativa y nuevas tecnologías. Barcelona: Praxis.

Graves, D. (1992). Estructurar un aula donde se lea y se escriba. Buenos Aires.

Jolibert, J. (1995). Formar niños lectores de textos. Santiago de Chile: Dolmen.

Litwin, E., Magio, M. \& Lipsman, M. (Comp.) (2005). Tecnologías en las aulas. Las nuevas tecnologías en las prácticas de la enseñanza. Casos para el análisis. Buenos Aires: Amorrortu Editores.

Machado, A.M. (1997). Lectura y nuevas tecnologías. Bogotá: Fundalectura.

Vásquez, G. (2000). Propuestas de alquimia escolar. Bogotá: Siglo XXI Editores. 